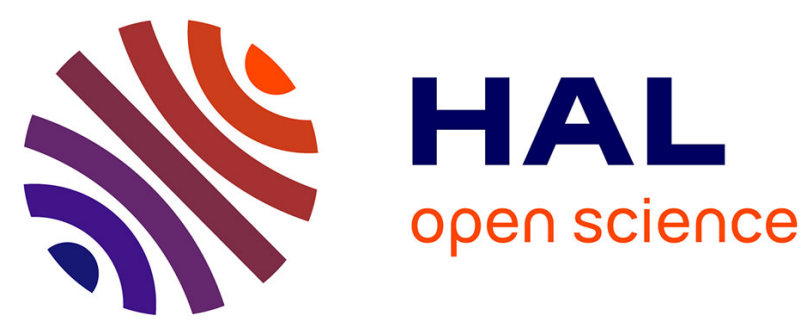

\title{
Introduction. Un encastrement social du commerce ?
}

Claude Didry, Florent Le Bot, Corine Maitte, Michela Barbot

\section{To cite this version:}

Claude Didry, Florent Le Bot, Corine Maitte, Michela Barbot. Introduction. Un encastrement social du commerce ?. L'Homme et la Société, 2021, n 211 (3), pp.15-26. 10.3917/lhs.211.0015 . halshs03506490

\section{HAL Id: halshs-03506490 \\ https://shs.hal.science/halshs-03506490}

Submitted on 2 Jan 2022

HAL is a multi-disciplinary open access archive for the deposit and dissemination of scientific research documents, whether they are published or not. The documents may come from teaching and research institutions in France or abroad, or from public or private research centers.
L'archive ouverte pluridisciplinaire HAL, est destinée au dépôt et à la diffusion de documents scientifiques de niveau recherche, publiés ou non, émanant des établissements d'enseignement et de recherche français ou étrangers, des laboratoires publics ou privés. 
Introduction

Un encastrement social du commerce?

\author{
Claude DIDRY \\ Centre Maurice Halbwachs (UMR 8097), CNRS, ENS, \\ EHESS
}

Florent LE BOT

IDHES $S^{1}$ (UMR 8533), $\mathrm{UEVE}^{2}, \mathrm{CNRS}$

Corine MAITTE

Laboratoire $\mathrm{ACP}^{3}$ (EA 3350), $\mathrm{UPEM}^{4}$

Michela BARBOT

IDHES $^{5}$ (UMR 8533) CNRS ENS Paris-Saclay

\footnotetext{
${ }^{1}$ Institution et Dynamiques Historiques de l'Économie

${ }^{2}$ Université d'Évry Val d'Essonne Paris-Saclay

${ }^{3}$ Analyse Comparée des Pouvoirs

${ }^{4}$ Université Paris Est Marne La Vallée

${ }^{5}$ Institution et Dynamiques Historiques de l'Économie
} 
La fin du salariat serait-elle en vue ? Un capitalisme plus «agile», ou plus «liquide» adviendrait-il? Les formes les plus contemporaines du capitalisme à travers notamment le travail de plateformes, mobilisant les travailleurs du clic (Casilli 2019), contribueraient-elles à cette transformation radicale? La situation présente serait censée marquer un tournant majeur, déterminé par la énième « révolution industrielle », celle de l'économie numérique et de la société digitale. Ce tournant est régulièrement invoqué depuis une vingtaine d'années afin de justifier les réformes du droit du travail, en prévoyant notamment le passage d'un statut de salarié à une position «d'entrepreneur de soi-même » (Didry et Le Bot 2015). Cette vision des derniers développements du capitalisme renvoie en fait à un récit couramment admis, faisant du rapport salarial le creuset du capitalisme. Ainsi, le salariat résulterait en particulier en France, du démantèlement de l'ordre corporatif par la Révolution. En GrandeBretagne, son émergence a longtemps été imputé à la libération de la maind'œuvre suscitée par les enclosures, jetant les paysans pauvres, privés de l'accès aux communs, dans les bras de l'industrie naissante (Chambers 1953). Ce salariat fondamentalement négatif (la fin des corporations, l'effondrement de la société rurale) est alors présenté comme le fondement d'une « révolution » dite « industrielle » rendue possible par l'exploitation d'une main-d'œuvre atomisée, ensuite relayée et intensifiée par la mécanisation des installations productives. Ce récit est marqué par la figure de l'expropriation - expropriation de la terre pour le petit paysan, expropriation des moyens de production pour l'artisan des corporations comme base originaire du salariat, et de l'exploitation, sous forme de la subordination salariale qui s'ensuivit.

Face à ce grand récit ancien, une historiographie plus récente éclaire le poids que représente l'ancrage rural des sociétés affectées par l'émergence du capitalisme, en remettant en cause la vision d'un « décollage » (d'un «take-off » à la Rostow) agro-industriel reposant sur la mécanisation. Loin du tableau mythifié d'une «révolution industrielle» (Jarrige 2015), s'esquisse la réalité d'un développement de la production initiée par une activité commerciale de plus en plus prospère. Le débat historiographique qui interroge les contours d'une «révolution industrieuse» (De Vries 2008) porte sur la part à attribuer dans cette dynamique à l'accroissement de la population, à l'augmentation du temps de travail et à son intensification, aux progrès technologiques et à l'élargissement de la 
consommation et des marchés, en proposant, qui plus est, de sortir du prisme hollando-centré et urbain de Jan de Vries (Béaur 2017). Par ailleurs, le rôle incitatif joué par les États est également mis en exergue. Ainsi, en France dès le XVII ${ }^{\mathrm{e}}$ siècle, les inspecteurs des manufactures institués par le colbertisme ont engagé une activité de recensement et d'encadrement de productions manufacturières diffuses dans les campagnes en s'appuyant sur une systématisation des règlements corporatifs urbains (Minard 1998, Minovez 2012). Cette diffusion de la production marchande dans les campagnes constitue l'un des aspects de ce que Franklin Mendels (1972), et bien d'autres, ont nommé la «protoindustrie ». Celle-ci est assimilée par Max Weber, dans l'Éthique protestante, à un «capitalisme traditionnaliste» dominé par des commerçants distribuant de l'ouvrage à domicile, tant dans les ateliers artisanaux des villes que dans les campagnes (putting-out system). De nombreux auteurs, comme Jean-Marc Olivier (2004) pour le Jura morézien, ont montré l'effet d'accumulation financière et technique que permet cette «industrialisation douce » à travers une succession de cycles productifs, là par exemple de la production de clous jusqu'à la fabrication de lunettes, en passant par celle d'horloges. Elle permet parfois de perpétuer un modèle d'organisation sociale du cultivateur propriétaire indépendant pluriactif (Villani 1990). Cette industrialisation différente par rapport à la grande concentration usinière présentée comme le modèle canonique de la «révolution industrielle » (on pourrait d'ailleurs parler d'une altero-industrialisation plutôt que d'une proto-industrialisation) adopte une configuration spécifique en Italie septentrionale marquée par un développement des activités marchandes dans le monde rural parallèlement à une "désindustrialisation » urbaine qui aurait commencé à partir du XVII ${ }^{\mathrm{e}}$ siècle (De Maddalena 1982 ; Dewerpe 1985 ; Cento Bull 1989). Les études sur les districts industriels (notamment Becattini 1987; Brusco 1989 ; Lescure 2006) et sur les « communautés territoriales d'entreprises » (Bagnasco 1999 ; Amatori et Colli 2001) montrent par ailleurs que, dans plusieurs contextes urbains, l'usine et l'atelier artisanal ont été deux formes organisationnelles fortement complémentaires et ont noué des relations constantes et parfois étroites avec la campagne environnante (Borruso 1997 ; Fontana 1997 ; Maitte 2001 ; Eck et Lescure 2002 ; Belfanti 2009; Maitte 2009). 
Loin de l'affirmation prométhéenne d'une bourgeoisie industrielle, le processus qui se dessine à travers ces différentes fresques part de l'affirmation progressive du commerce, de la geste marchande, qui s'insinue comme activité économique dans les institutions existantes, qu'il s'agisse des familles rurales ou des corporations urbaines. Les activités productives liées au commerce assurent ainsi un complément de revenus aux populations paysannes; loin de la vision d'un exode massif par expropriation, elles contribuent à fixer celles-ci dans le monde rural, tout en leur ouvrant, dans le meilleur des cas, l'accès à de nouvelles formes de consommation et en rendant possible le paiement des impôts. Elles mobilisent également les institutions corporatives dans les villes, tout à la fois dans leur fonction de contrôle des productions rurales, de coordination de l'activité menée dans les ateliers (Arrighetti et Seravalli 1999; Belfanti 2002) et de discipline des maîtres et compagnons urbains. La production de marchandises - loin de se concentrer dans de grandes installations s'inscrit dans l'espace domestique intégrant les membres de la famille, jusqu'à de véritables ateliers domestiques attirant des ouvriers du voisinage. Elle suscite une conflictualité sociale que l'on retrouve depuis les révoltes des artisans drapiers en Flandre ou en Italie au Moyen-Âge (Milhaud 1897 ; Stella, 1993 ; Franceschi, 1993) jusqu'à celles des canuts lyonnais tout au long des $\mathrm{XVIII}^{\mathrm{e}}$ et $\mathrm{XIX}^{\mathrm{e}}$ siècles (Hupfel 2010). Cette situation se traduit également par des formes d'entreprises ouvrières, qui, en France, se lisent dans le louage d'ouvrage défini par les articles 1779 à 1799 du Code civil, en déclarant l'ouvrier rémunéré à la pièce « entrepreneur en la partie qu'il traite » (art. 1799). Comme enchaînement de louages d'ouvrage, le marchandage correspond à une sous-traitance en cascade multipliant les intermédiaires, sans que l'on ne sache finalement « où s'arrête le patron et où commence l'ouvrier » (Gaillard 1997 [1976]).

Le dossier que nous présentons vise à éclairer cet encastrement de la production marchande dans l'univers familial, en interrogeant tout à la fois la place de la famille, celle des corporations, ainsi que les porosités de la ville et de la campagne dans une perspective de diffusion, de circulation et d'interdépendance économique et sociale. Il est issu d'un programme de recherche ambitieux destiné à éclairer ces dynamiques dans les pays d'Europe méridionale que sont l'Espagne, la France et l'Italie, au regard de 
chemins bien balisés s'agissant de l'Europe du nord, et en adoptant un regard sur la longue durée 6 .

\section{Un encastrement familial des activités commerciales}

Comme le montre Mathieu Scherman en discutant les thèses de Fernand Braudel (1985) et d'autres auteurs (notamment Alfani 2006), la famille représente pour le capitalisme des cités-États italiennes la base d'un système d'alliance permettant l'affirmation, à une échelle internationale, d'une activité commerciale et bancaire qui s'étend vers l'Europe du Nord, à travers son implantation londonienne. Elle se présente également comme le cadre de stratégies d'ascensions sociales pour des cités moins fortunées telle que Trévise, via l'investissement dans des activités productives qui conduisent parfois à la réussite économique de la première génération. $\mathrm{La}$ deuxième génération peut alors se tourner vers des activités plus intellectuelles, comme le notariat, avant que la troisième génération ne puisse vivre de ses rentes et espérer se retirer à la campagne. Dans le même temps, le développement d'une activité marchande fournit à l'historien la matière de ses analyses, à travers les sources comptables et fiscales.

Sans aller jusqu'aux réussites les plus marquantes que donne à voir cette trajectoire sociale idéale dans l'Italie de la Renaissance, la famille constitue également la base d'activités productives à domicile en favorisant leur reproduction et leur ancrage dans des espaces géographiques spécifiques, à des époques plus récentes (Colli 2003; Cavaciocchi 2009; Boudjaaba 2014). Cela se retrouve notamment dans le cas des tisserands de Manresa analysé par Lluis Viros, durant la séquence 1890-1920. La période se caractérise certes par le développement de grands établissements mécanisés qui coexistent alors, dans ce bourg bientôt annexé à l'agglomération barcelonaise, avec une part importante de production à domicile. Mais il faut compter ici avec une structure sociale complexe, dans laquelle les

${ }^{6}$ Programme « Mondes et marchés du travail dans l'Europe du Sud. Capabilités, vulnérabilités individuelles, familiales et collectives $\left(\mathrm{XV}^{\mathrm{e}}-\mathrm{XXI}^{\mathrm{e}}\right.$ siècles ; Espagne, France, Italie, Portugal, Suisse Romande et italienne), soutenu par l'École des hautes études hispaniques et ibériques (Casa de Velázquez) et coordonné par l'IDHES ENS de Cachan (Florent Le Bot), 2013-2016. 
négociants se trouvent placés en position de donneurs d'ordres, tant à l'égard de chefs d'atelier faisant travailler à domicile les membres de leur famille et les ouvriers qu'ils embauchent parfois, que de chefs d'équipe qui recrutent dans l'usine les membres de leur équipe sur une base d'interconnaissance. La rémunération à la pièce est la règle générale, conduisant le chef d'atelier ou d'équipe à payer à leur tour - à la pièce ou au temps- ceux qui travaillent avec lui. Cette structuration sociale tranche alors avec un partage net en deux classes, ouvriers et propriétaires des moyens de production, que tentent alors de faire prévaloir les organisations syndicales.

Bien que dans des dimensions plus modestes, le partage entre production artisanale à domicile et manufactures marquées par une certaine division des tâches se retrouve dans le cas de la lutherie au sein de la région de Mirecourt. L'inculcation familiale ou par apprentissage dans un atelier voisin s'affirme ici, selon Hélène Claudot-Hawad, essentielle dans la transmission du métier au cours de la première moitié du $\mathrm{XX}^{\mathrm{e}}$ siècle, en partage cependant avec l'enseignement professionnel. Si la prospérité de l'immédiat après-guerre fait apparaître la lutherie comme un débouché «naturel» pour les jeunes, les difficultés des années 1930, qui se prolongent au lendemain de la Seconde Guerre mondiale, rendent la transmission plus problématique: elles en font une gageure pour les familles, avant que cette spécialité finalement ne s'étiole.

Dans le cas des portefaix du port de Barcelone, les faquines, présenté par Juanjo Romero, nous serions tentés de penser que l'activité professionnelle offre peu de savoir-faire à transmettre. Mais le poids de la famille se manifeste ici à travers le nombre réduit de patronymes relevé dans les archives portuaires, en révélant une stratégie d'alliances proche de l'endogamie. L'enjeu est alors celui du contrôle social, tout à la fois comme barrière à l'entrée pour éviter l'afflux de main-d'œuvre extérieure au cercle des portefaix et comme transmission de valeurs. En effet, l'honnêteté dans le débardage et la livraison des colis va devenir un argument de poids pour maintenir les contours d'un groupe social que la remise en cause du régime corporatif tend à ébranler. 


\section{Un autre regard sur les corporations}

La diffusion des activités marchandes dans des sociétés majoritairement rurales conduit à s'interroger sur le rôle des corporations dans le processus de développement du capitalisme. Selon une historiographie classique, les corporations symbolisent le traditionalisme des métiers dans la société d'Ancien Régime. Cette connotation négative se retrouve dans la dénonciation libérale des «corporatismes » que l'on retrouve à partir du $\mathrm{XVIII}^{\mathrm{e}}$ siècle et qui se développe ensuite pour critiquer le poids du syndicalisme dans certaines branches ou le refus du changement face à des politiques dites «de modernisation» (Kaplan et Minard 2004). Des travaux plus récents ont conduit cependant à remettre en cause le caractère systématique des régulations corporatives, en mettant en évidence des situations marquées par l'absence de cadres corporatifs, comme dans le cas du bâtiment pour certaines villes médiévales (Bernardi 2009). Cela a pu conduire à envisager l'existence précoce de salariés jusque dans les villes et les campagnes médiévales (Beck, Bernardi et Feller 2014). Mais, le terme de salariat est sans doute excessif pour saisir des formes très variées de travail rémunéré qu'une partie des acteurs appréhendent moins sous l'angle d'un contrôle de leur activité individuelle par un employeur, que sous celui d'une sous-traitance assumée sur le mode de l'indépendance artisanale et de la petite entreprise (Didry 2016).

Ainsi, sans aller jusqu'à nier le rôle des corporations dans une perspective de longue durée, le poids des activités rurales suggère de revenir sur le rôle de ces institutions en sortant d'une focalisation sur l'exercice d'un contrôle disciplinaire du travail. Dans le sillage d'une série de travaux qui interrogent à nouveaux frais le rôle économique - voire entrepreneurial des corporations (Epstein and Prack 2008; De Munck 2011, 2018 ; Caracausi 2017), les articles présentés dans ce dossier permettent ainsi d'appréhender les corporations en les rapportant à des configurations spécifiques, variant en fonction des lieux et des périodes. Dans les villes italiennes de la Renaissance, Mathieu Scherman aborde les corporations, sans s'y attarder, comme des points de passage dans des stratégies familiales. Elles hébergent ainsi les activités qui vont devenir le support d'une accumulation primitive dans la recherche d'une ascension sociale sur plusieurs générations, en montrant que la corporation n'a rien d'une 
« caste » enfermant ses membres dans une reproduction héréditaire. On pourrait parler ici d'un usage opportuniste des corporations, dans une dynamique familiale d'ascension sociale.

Dans la Rome du XVII ${ }^{\mathrm{e}}$ siècle, le mouvement des boutiques et la circulation des outils indiquent une forme de débordement des corporations, qui peinent à contrôler les activités dont elles ont la charge. Les règlements corporatifs tentent pourtant de trouver une parade à cette situation sans véritablement y parvenir, comme le montre Eleonora Canepari qui met en exergue un régime de corporation absente, neutralisée en quelque sorte par le mouvement de ventes et de locations des outils et des boutiques.

De manière très différente - et en un sens plus conforme à la vision classique -, dans le cas des portefaix barcelonais, la corporation est la base d'une affirmation du groupe, identifié à des qualifications et à des valeurs tout en régulant l'accès des étrangers. Appréhendée par Juan Romero tandis qu'elle est remise en cause par les percées libérales qui traversent la société espagnole et sa monarchie, la corporation est le lieu de coagulation d'un véritable culte de la profession qui se prolonge - au-delà de sa suppression - dans la création de sociétés mutuelles reposant sur les valeurs et le contrôle social caractéristique de l'institution corporative. Elle se situe alors dans ce que l'on est tenté de nommer - à la manière de Pierre Bourdieu - un « champ » corporatif dans lequel la corporation des faquines joue son avenir par des alliances avec les corporations voisines (charretiers et muletiers), tout en assumant sa compétition avec celles-ci, notamment quand ses dernières cèdent à la tentation de s'assimiler à des entreprises. La corporation est ici une institution revendiquée, quitte à la retraduire dans le langage associatif qui sied à une époque d'ouverture au libéralisme.

Enfin, dans le cas des tisserands barcelonais de Manresa, la corporation tend à se dissoudre dans un univers productif soumis aux aléas du marché et conduit, pour y faire face, à des aménagements tel que, par exemple, le passage de la soie au coton dans le tissage des rubans étroits. Dans ce contexte, l'héritage corporatif tend à s'effacer face à l'implantation d'établissements industriels (travail réuni) qui ouvrent l'activité aux femmes par une division plus importante du travail et un soi-disant allègement de la charge physique résultant de la mécanisation, qui vise à 
rendre le travail des femmes acceptable dans une société présupposant la supériorité physique des hommes sur les femmes. Cet héritage se réduit alors à un tarif déterminant le prix des pièces, pour coordonner les établissements mécanisés et les ateliers domestiques.

\section{Les porosités entre villes et campagnes}

La tension entre les villes et les campagnes ne se résout pas nécessairement par une spécialisation entre des villes orientées vers une production industrielle de plus en plus concentrée et des campagnes vidées de toute activité artisanale, au contraire (Terrier 1996 ; Epstein 2011; Minovez, Verna et Hilaire-Pérez 2013). Dans les villes italiennes de la Renaissance analysées par Mathieu Scherman, la campagne demeure le point d'aboutissement d'une stratégie familiale d'ascension sociale à travers la constitution d'un domaine dans lequel les membres de la famille aspirent à se retirer.

Dans le cas barcelonais, la partition entre ville et campagne est soumise à une évolution liée à un développement économique se traduisant par un débordement de la métropole sur les villages voisins. Les faquines barcelonais étudiés par Juan Romero se regroupent dans le village de Gracia, avant que celui-ci ne devienne un quartier de la capitale catalane. Il en va de même, dans l'article de Luis Viros, pour les rubaniers de Manresa, village finalement annexé à l'agglomération barcelonaise.

La ruralité des luthiers de Mirecourt et sa région peut apparaître, à certains égards, comme la base d'une maîtrise familiale de la transmission des savoir-faire et du maintien d'une production domestique. Mais elle tient probablement à un éloignement des centres urbains, que renforcent les évolutions de la frontière au lendemain de la guerre de 1870. Cela se traduit par des relations avec plusieurs agglomérations (Paris, Nancy), qui tiennent soit à l'existence de sociétés commerciales dotées de plusieurs implantations, soit aux réseaux de correspondances entre les artisans de Mirecourt et des boutiquiers nancéiens ou parisiens. 
La lecture des développements économiques et sociaux analysés dans les articles de ce dossier suggère de saisir l'émergence du capitalisme en sortant du modèle classique du désencastrement social des activités économiques proposé par Karl Polanyi, à partir d'une modélisation de dynamiques rapportées dans le cas britannique, au mécanisme de l'expropriation censé conduire à un marché autorégulé. S'inscrivant dans une perspective d'analyse qui invite à sortir d'une démarche en termes de retard par rapport à une Angleterre idéalisée a posteriori, cet ensemble d'articles engagent à envisager une mutation plus profonde - voire souterraine - de sociétés soumises à une intensification des échanges commerciaux. De ce point de vue, le détour par des pays d'Europe méridionale présente l'intérêt de saisir la manière dont les transactions commerciales s'insinuent dans des stratégies familiales et mobilisent, sans nécessairement les détruire, des institutions telles que les corporations. Face aux remises en cause actuelles du salariat et à la soi-disant libération que constituerait - face à la «subordination» salariale l'autoentrepreneuriat suscité par le développement du «numérique » et des plateformes, ces articles suggèrent également que le salariat lui-même est une institution située historiquement, émergeant plus tardivement d'une production marchande d'abord dispersée dans des ateliers domestiques. Ainsi, le culte de l'autoentrepreneuriat et le fétichisme du numérique, comme grand remplacement du travail par des robots, mériteraient d'être rapportés à ces situations anciennes, afin de sortir d'un certain enchantement par le progrès technologique, et pour y voir l'indice d'une crise sociale profonde, ouvrant sur une crise démocratique, liées à une invisibilisation du travail.

\section{Références bibliographiques}

ALFANI Guido (s.d.), 2006. «Il ruolo economico della famiglia », numéro spécial de Cheiron 45-46.

ARrighetTI Gilberto et SERAVAlli Alessandro (s.d.), 1999. Istituzioni intermedie e sviluppo locale, Rome, Donzelli. 
AmATORI Franco et Colli Andrea (s.d.), 2001. Comunità di imprese. Sistemi locali in Italia tra Otto e Novecento, Bologne, Il Mulino.

BAGNASCO Arnaldo, 1999. «La città come società locale », id., Tracce di comunità, Bologne, Il Mulino.

BEAUR Gérard, 2017. "La révolution industrieuse en débat ", Revue d'histoire moderne et contemporaine, $4, \mathrm{n}^{\circ}$ 64-4, p. 7-24.

BeCATtini Giorgio (s.d.), 1987. Mercato e forze locali : il distretto industriale, Bologne, Il Mulino.

BeLfanti Carlo Marco, 2009. «The genesis of a hybrid: early industrial districts between craft culture and factory training ", dans Giacomo Becattini, Marco Bellandi, Lisa de Propris (s.d.), A Handbook of Industrial Districts, Cheltenham, Edward Elgar, p. 10-17.

Belfanti Carlo Marco, 2002. "Market and social institutions at the origins of some Italian industrial districts », dans F. DE GOEY, J. W. VELUWENKAMP (s.d.), Entrepreneurs and Institutions in Europe and Asia, Amsterdam, Aksant Academic Publishers, p. 257-270.

BeCK Patrice, BERnARDI Philippe, FeLLER Laurent, 2014. Rémunérer le travail au Moyen Âge. Pour une histoire sociale du salariat, Paris, Picard.

BERnARDI Philippe, 2009. Maître, valet et apprenti au Moyen Âge. Essai sur une production bien ordonnée, Toulouse, CNRS/Université de Toulouse-Le Mirail.

BOUDJAABA Fabrice (dir.), 2014. Le travail et la famille en milieu rural, $X V I^{e}-X X I^{e}$ siècle, Rennes, Presses universitaires de Rennes.

BORRUSO Edoardo, 1997. «Dal laboratorio artigiano alla piccola impresa urbana », dans BelfaNTI Carlo Marco et MACCABELli Terenzio (s.d.), Un paradigma per i distretti industriali, Brescia, Grafo, p. 71-93. 
BRUSCO Sebastiano, 1989. Piccole imprese e distretti industriali, Turin, Rosenberg e Sellier.

CARACAUSI Andrea, 2017, « Information asymmetries and craft guilds in pre-modern markets: evidence from Italian proto-industry », The Economic History Review, 70, p. 397-422.

CASILLI Antonio, 2019. En attendant les robots. Enquête sur le travail du clic, Paris, éd. du Seuil.

CAVACIOCCHI Simonetta (s.d.), 2009. La famiglia nell'economia europea secoli XIII-XVIII / The Economic Role of the Family in the European Economy from the 13th to the 18th Centuries, Firenze, Firenze University Press.

Cento Bull Anna, 1989. «Proto-industralization, Small-scale Capital Accumulation and Diffused Entrepreneurship: the Case of the Brianza in Lombardy (1860-1950) », Social History, 1989/2, p. 177-200.

Chambers Jonathan D., 1953. «Enclosure and labour supply in the industrial revolution », Economic History Review, 5/3, p. 319-343.

Colli Andrea, 2003. The History of Family Business 1850-2000, Cambridge, Cambridge University Press.

DE MuncK Bert, 2018. Guilds, Labour and the Urban Body Politic: Fabricating Community in the Southern Netherlands, 1300-1800, New York, Routledge.

DE MunCK Bert, 2011. «Gilding golden ages. Perspectives from early modern Antwerp on the guild-debate, c. 1450- c. $1650 »$, European Review of Economic History, 2011 / 15, pp. 221-253.

De MadDalena Aldo, 1982. Dalla città al borgo. Avvio di una metamorfosi economica e sociale nella Lombardia spagnola, Milan, Franco Angeli. 
DE VRIES Jan, 2008. The Industrious Revolution. Consumer Behavior and the Household Economy, 1650 to the Present. Cambridge, Cambridge University Press.

DEWERPE Alain, 1985. L'Industrie aux champs: essai sur la protoindustrialisation en Italie septentrionale (1800-1880), Rome, École française de Rome.

DIDRY Claude, 2016. L'institution du travail. Droit et salariat dans l'histoire, Paris, La Dispute.

DIDRY Claude et LE BOT Florent, 2015. «Un dépassement capitaliste du salariat? Une sociohistoire en trois actes et impasses. », L'Homme et la société, $\mathrm{n}^{\circ} 195-196$, p. 51-72.

ECK Jean-François et LESCURE Michel (éd.), 2002. Villes et districts industriels en Europe occidentale (XVII ${ }^{e}-X X^{e}$ siècle), Tours, Presses universitaires François Rabelais.

EPSTEIN Stephan R. (s.d.), 2001. Town and country in Europe, 13001800, Cambridge, Cambridge Universty Press.

EPSTEIN Stephan R. et PRAK Marteen (s.d.), 2008. Guilds, Innovation, and the European Economy, 1400-1800, Cambridge, Cambridge University Press.

FONTANA Gian Luigi (s.d.), 1997. Le vie dell'industrializzazione europea: sistemi a confronto, Bologne, Il Mulino.

FRANCESCHI Franco, 1993. Oltre il "tumulto $»:$ i lavoratori fiorentini dell'arte della lana fra Tre e Quattrocento, Firenze, L. S. Olschki.

GaILlard Jeanne, 1997 [1976]. Paris, la Ville (1852-1870), Paris, L'Harmattan. 
HUPFEL Simon, 2010. «L'évolution comparée des manufactures de soieries de Londres et de Lyon, 1789-1848 : une approche institutionnaliste », thèse pour le doctorat de science économique, ENS Lyon.

JARRIGE François, 2015. "Révolutions industrielles : histoire d'un mythe », Revue Projet 2015/6 (n³49), p. 14-21.

KAPLAN Steve et MinARD Philippe (s.d.), 2004. La France, malade du corporatisme? XVIII ${ }^{e} X X^{e}$ siècles, Paris, Belin, 2004.

LESCURE Michel (s.d.), 2006. La mobilisation du territoire. Les districts industriels en Europe occidentale du XVIIe au XXe siècles, Paris, CHEFF.

MAITTE Corine, 2001. La trame incertaine, le monde textile de Prato aux XVIII ${ }^{e}$ et XIX ${ }^{e}$ siècles, Villeneuve $\mathrm{d}$ 'Ascq, Presses universitaires du Septentrion.

MAITTE Corine, 2009. «Identity of products and identity of industrial districts », Giacomo Becattini, Marco Bellandi, Lisa De PROPRIS (s.d), The Handbook on Industrial Districts, E. Elgar publisher, vol.1: Origin and theories, p. 18-31.

MENDELS Franklin, 1972. «Proto-industrialization: the first phase of industrialization process », Journal of Economic History, 32, p. 241-261.

MiLHAUd Albert, 1897. «La lutte des classes en Flandres au MoyenAge ; artisans contre marchands ", Revue internationale de sociologie 5/2, p. 111-128.

MINARD Philippe, 1998. La fortune du colbertisme. État et industrie dans la France des Lumières, Paris, Fayard.

MinOveZ Jean-Michel, 2012. La puissance du Midi. Les draperies de Colbert à la Révolution, Rennes, Presses universitaires de Rennes. 
Minovez Jean-Michel, Verna Catherine, HilaIRE-Perez Liliane (dir.), 2013. Les industries rurales dans l'Europe médiévale et moderne, Toulouse, Presses universitaires du Mirail.

OLIVIER Jean-Marc, 2004. Des clous, des horloges et des lunettes. Les campagnards moréziens en industrie (1780-1914), Paris, CTHS.

STElla Alessandro, 1993. La révolte des Ciompi. Les hommes, les lieux, le travail, Paris, éd. de l'EHESS.

TERRIER Didier, 1996. Les deux âges de la protoindustrie. Les tisserands des villages du Cambrésis et du Saint-Quentinois, 1730-1880, Paris, éd. de l'EHESS.

VILLANI Pasquale (s.d.), 1990. La pluriattività negli spazi rurali : ricerche a confronto, Bologne, Istituto Alcide Cervi. 